\title{
Adherence to the 2008 IHS guidelines for controlled trials of drugs for the preventive treatment of chronic migraine in adults
}

Cephalalgia

2019, Vol. 39(8) 1058-1066

(C) International Headache Society 2019

Article reuse guidelines:

sagepub.com/journals-permissions DOI: |0.1 I77/0333 I024|984775 |

journals.sagepub.com/home/cep

@SAGE

\author{
Marie Deen ${ }^{1} *$ (D), Daniele Martinelli ${ }^{2,3},{ }^{*}$, Judith Pijpers ${ }^{4}$, \\ Hans-Christoph Diener ${ }^{5}$, Stephen Silberstein ${ }^{6}$, \\ Michel D Ferrari ${ }^{4}$, Messoud Ashina', Cristina Tassorelli ${ }^{2,3} \mathbb{( D}$ and \\ Hsiangkuo Yuan'
}

\begin{abstract}
Introduction: Since the definition of chronic migraine as a new disease entity in 2004, numerous clinical trials have examined the efficacy of preventive treatments in chronic migraine. Our aim was to assess the adherence of these trials to the Guidelines of the International Headache Society published in 2008.

Methods: We searched PubMed for controlled clinical trials investigating preventive treatment for chronic migraine in adults designed after the release of the Guidelines and published until December 20I7. Trial quality was evaluated with a I3-item scoring system enlisting essential recommendations adapted from the Guidelines.

Results: Out of 3352 retrieved records, we included 16 papers in the analysis dealing with pharmacological treatment of chronic migraine. The median score was 6.5 (range 2-13). All trials were randomized, the large majority (8I.25\%) were placebo-controlled and double-blinded (87.5\%). Adherence was lowest on i) a priori definition of outcomes (31.25\%), ii) primary endpoint definition (37.5\%\%) and iii) trial registration $(37.5 \%)$.

Discussion: Most clinical trials adhered to the recommendations of the IHS, whereas adherence to migraine-specific recommendations was lower. Greater awareness and adherence to the guidelines are essential to improve the quality of clinical trials, validity of publications and the generalizability of the results.
\end{abstract}

\section{Keywords}

Methodology, clinical trials, guidelines, chronic migraine, headache

Date received: 23 September 2018; revised: 22 February 2019; accepted: 22 March 2019

\section{Introduction}

Clinical trials are expensive and resource demanding. This is especially true for CNS disorders, such as migraine, which often impose higher development costs and have a lower success rate compared to drugs targeting other disorders (1). To enhance the quality and validity of clinical trials, and to enable a comparison between different compounds, it is important to ensure reliability and consistency in trial design and outcome parameters. For this purpose, a set of recommendations for controlled trials on preventive treatment of chronic migraine $(\mathrm{CM})$ (according to the ICHD-2 chronic migraine revised version (CM-R) criteria (2)) in adults was published by the Task Force of

\footnotetext{
'Danish Headache Center, Department of Neurology, Rigshospitalet Glostrup, Denmark

${ }^{2}$ Headache Science Center, IRCCS C. Mondino Foundation, Pavia, Italy ${ }^{3}$ Department of Brain and Behavioral Sciences, University of Pavia, Pavia, Italy

${ }^{4}$ Department of Neurology, Leiden University Medical Center, Leiden, the Netherlands

${ }^{5}$ Medical Faculty of the University Duisburg-Essen, Essen Germany ${ }^{6}$ Department of Neurology, Thomas Jefferson University Hospital, Philadelphia, PA, USA

*These authors contributed equally to this work.
}

\section{Corresponding author:}

Daniele Martinelli, Headache Science Center, IRCCS C. Mondino Foundation, Via Mondino 2, 27I00 Pavia, Italy.

Email: daniele.martinelli@mondino.it 
the International Headache Society (IHS) in 2008 (3). Similar but separate guidelines had previously been published for episodic migraine, tension-type headache and cluster headache (4-6) - all with the purpose of strengthening the quality of the trials in order to generate evidence-based treatment recommendations for different headache disorders.

The 2008 Guidelines for trials on preventive treatment of CM offered recommendations on subject selection, trial design, outcome evaluation, statistical analysis, and publication. Recently, an updated edition of the Guidelines was published (7). These were guided by a critical analysis of RCTs published since 2008 . The information and knowledge gathered during this critical analysis are here illustrated for the reader's perusal. More precisely, the objective of this study was to quantitatively assess the adherence of the published trials to the Guidelines of the International Headache Society published in 2008 with the specific aim of identifying critical areas to be addressed.

\section{Methods}

\section{Eligibility criteria}

Clinical trials on preventive treatments for CM published between May 2008 and December 2017.

Specific inclusion criteria were: a) Trials on adult $\mathrm{CM}$ patients; b) investigation of preventive treatments: oral or injected drugs, including nerve blocks; c) controlled trials; d) study design developed after the publication of the 2008 Guidelines and enrolment started consequently.

Trials published after 2008 but started anteriorly in terms of design and/or enrolment were excluded. Trials on acupuncture, behavioral interventions and devices were excluded.

\section{Search method}

We searched the PubMed database for articles on clinical trials and clinical studies on the preventive treatments for CM published between 1 May 2008 and 31 December 2017 without language restrictions, using the terms "((migraine) OR (headache)) AND ((randomized controlled trial) OR (randomized) OR (placebo) OR (drug therapy) OR (randomly) OR (trial) OR (preventive) OR (group) NOT (animals) NOT ((humans) and (animals))".

\section{Screening of papers}

Titles and abstracts of studies identified by the literature search were screened for eligibility. If title and abstract evaluation was deemed not sufficient to determine eligibility (or exclusion), the manuscript was evaluated in full.

\section{Data analysis}

Trials were evaluated according to a 13-item scoring system based on the recommendations listed in the 2008 Guidelines for obtaining a high-quality trial (Table 1). A similar scoring system has been applied previously for assessing clinical trials in EM (8). For the scoring purpose, the definition of chronic migraine used was the revised version (CM-R) published in 2006 (2); patients with medication overuse headache $(\mathrm{MOH})$ were allowed but only if the study population was stratified accordingly. The assessed trials received 1 point for each item of the scoring system they adhered to, which resulted in a score ranging from 0 (no recommendation applied) to 13 (the entire set of recommendations was applied). Four investigators (MD, DM, SY and JP) performed the search of the database and the evaluation of the papers. All trials were first evaluated by one investigator. Subsequently, all scorings were reviewed independently by two additional investigators with an interrater agreement above $90 \%$. In case of disagreement or incomplete agreement, this was resolved by discussion.

\section{Results}

\section{Inclusion of papers}

The search was performed on 31 December 2017 and yielded 3352 PubMed records. Out of the 3352 titles and abstracts screened, we identified 61 papers that satisfied the eligibility criteria and were therefore evaluated in full. Of these, we excluded 30: 26 because they evaluated the efficacy of interventions not covered in this review (devices in 16 papers, acupuncture in five, behavioral therapy in two, primary care intervention in two and stem cells in one case report); four studies were excluded because they dealt with chronic daily headache and not specifically with CM. Two additional studies were not retrievable or withdrawn by the editor. The remaining 29 papers were included in the full review. Of these, 11 studies reported data of trials that were devised and conducted before the publication of the 2008 Guidelines; in two additional studies it was not possible to define the temporal sequence with respect to the Guidelines. The final set that was included in the analysis was therefore formed by 16 studies (Figure 1).

\section{Evaluation of trials}

Of the total of 16 reviewed studies, $13(81 \%)$ were randomized, double blinded, placebo-controlled 
Table I. Scoring frame used for evaluating clinical trials on chronic migraine: For each item, we assigned a score of I if the recommendation was followed, 0 if it was not.

Selection of patients

I. Diagnostic criteria are according to the ICHD-2 CM-R.

2. Duration of observation is I month/28 days prospective using a detailed headache diary.

3. Monotherapy with wash-out or add-on therapy with stable preventive.

Trial design

4. Double-blind.

5. Placebo-controlled.

6. Randomization at trial entry.

7. Duration of treatment is at least 3 months.

8. Medication overuse stratified or not allowed.

Evaluation of results

9. The primary outcome is one of the following: Number of headache days with moderate or severe intensity. Number of migraine or probable migraine days. Number of migraine episodes.

10. The secondary outcomes included at least two of the following: Number of headache days with moderate or severe intensity. Number of migraine days. Number of migraine episodes (if not used as primary outcome). Intensity of headache (categorical mild/moderate/severe). Headache duration in hours. Responder rates $(\geq 30 \% / 50 \% / 25 \% / 75 \%$ reduction in number of headache days with moderate to severe intensity or migraine episodes). Acute treatment utilization. Conversion to episodic migraine.

II. At least one outcome on quality of life or disability.

12. NCT Registered before study enrollment.

13. Outcomes are specified a priori.

Min score $=0$, Max score $=13$

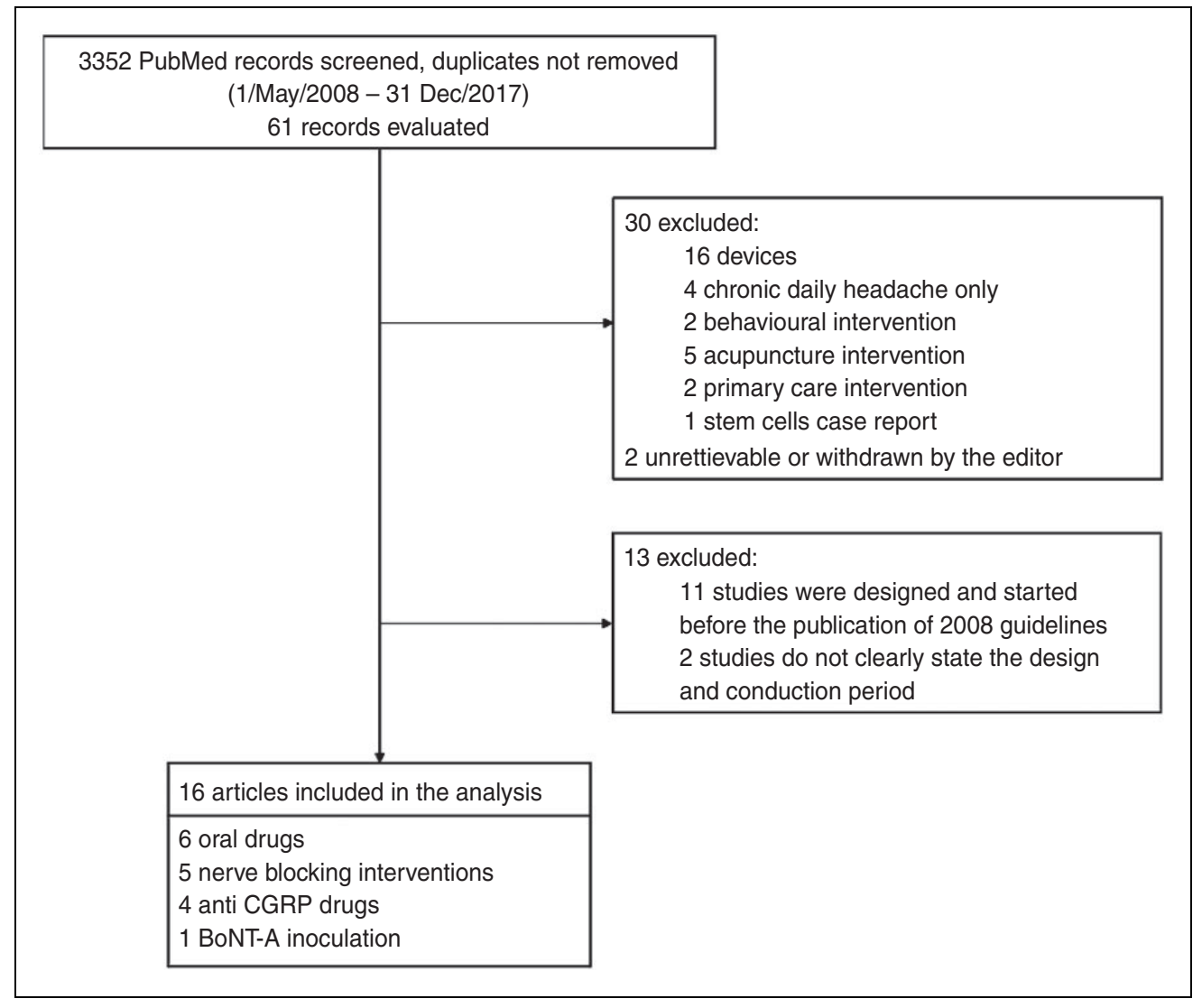

Figure I. Flowchart describing literature evaluation and paper selection.

CGRP: Calcitonin gene-related peptide; BoNT-A: botulinum toxin A). 
Table 2. Total scores attributed to the trials.

\begin{tabular}{|c|c|c|c|}
\hline Authors, Journal & Intervention & Year & $\begin{array}{l}\text { Total score } \\
(\min =0, \\
\max =13)\end{array}$ \\
\hline \multicolumn{4}{|l|}{ Trials dealing with oral or injected drugs } \\
\hline Silberstein et al., Neurology & Propranolol added to topiramate vs. placebo & 2012 & 13 \\
\hline Kwok et al., Pain Res & Ibudilast vs. placebo & 2016 & 12 \\
\hline Tepper et al., Lancet Neurol & Erenumab vs. placebo & 2017 & 12 \\
\hline Bigal et al., Lancet Neurol & TEV-48I 25 (fremanezumab) vs. placebo & 2015 & 10 \\
\hline Bigal et al., Neurology & TEV-48I 25 (fremanezumab) vs. placebo & 2016 & 10 \\
\hline Silberstein et al., $N$ Engl J Med* & TEV-48I 25 (fremanezumab) vs. placebo & 2017 & 10 \\
\hline Song et al., Eur Rev Med Pharmacol Sci & BonT-A and infrared polarized light & 2015 & 8 \\
\hline Ebrahimi-Monfared et al., Restor Neurol Neurosci & Melatonin vs. valproic acid & 2017 & 7 \\
\hline Lai et al., Acta Neurol Scand & Flunarizine vs. topiramate & 2017 & 6 \\
\hline Kashipazha et al., Curr Clin Pharmacol & Levetiracetam vs. sodium valproate & 2017 & 5 \\
\hline Soares et al., Nutr Neurosci. & OPFA $\omega-3$ vs. placebo & 2017 & 3 \\
\hline \multicolumn{4}{|l|}{ Trials dealing with nerve blocks } \\
\hline Inan et al., Acta Neurol Scand. & GON block vs. placebo & 2015 & 6 \\
\hline Palamar et al., Pain Phys & GON block vs. placebo & 2015 & 5 \\
\hline Dilli et al., Cephalalgia & GON block vs. placebo & 2015 & 5 \\
\hline Gul et al., Acta Neurol Scand & GON block vs. placebo & 2016 & 5 \\
\hline Cuadrado et al., Cephalalgia & GON block vs. placebo & 2016 & 2 \\
\hline
\end{tabular}

*Add on to trial in episodic migraine. Registered under the same NCT number.

GON: Greater Occipital Nerve.

parallel-arm trials; none had a cross-over design. Six studies evaluated the effect of oral medications (9-14), five the effect of injected medications (CGRP monoclonal antibodies in four cases (15-18) and botulinum toxin in one (19)), and five trials dealt with nerve blocks (20-24).

The scores attributed to the trials included in the evaluation are illustrated in Table 2. The median score was 6.5 , with a range of $2-13$. It is worth noting that the mean score of the studies dealing with oral and/ or injected drugs was higher (10, with a range of 3-13) than the score of trials dealing with nerve blocks $(5$, with a range of 2-6) (Figure 2).

The recommendations that were most frequently followed were: i) randomization $(100 \%)$, ii) double-blind design $(87.5 \%)$, and iii) placebo-controlled design $(81.25 \%)$. The three studies that were not placebo-controlled used an active comparator. Fifty percent of the trials applied the ICHD-2 CM-R diagnostic criteria as recommended. The recommendations that fewest trials followed were: i) The a priori definition of outcomes $(31.25 \%$ of the trials); ii) the primary endpoints being either number of headache days with moderate or severe intensity, number of migraine or probable migraine days or number of migraine episodes $(37.5 \%$ of the trials); iii) NCT registration before the recruitment $(37.5 \%$ of the trials); iv) duration of treatment period being three months (37.5\% of the trials); v) inclusion of healthcare outcomes/Quality of Life assessments as secondary endpoints (37.5\% of the trials).

As regards ii), primary endpoints, we found a wide variety of definitions. For example, several trials reported the number of all headache days instead of the number of moderate-to-severe headache days; in other cases, the intensity of pain was considered, but it was rated according to a visual analogue scale instead of using the recommended categorical scale ranging from 0 to 4 .

The recommendation regarding the 3-month duration of treatment was lowered by the nerve block studies $(n=5)$, which generally had a shorter treatment duration (1-4 weeks). However, it is worth noting that even after excluding these studies, the percentage of trials using a 3-month treatment period only increased to $45.5 \%$.

More than half of the studies $(62.5 \%)$ followed the recommendation on concomitant drug use (i.e. monotherapy with a 28-day washout period or add-on therapy with stable doses for other preventives for at least 3 months prior to baseline). The most common reasons for scoring 0 for this specific item in our scoring system were inadequate information on wash-out period or the period of being on stable doses before inclusion being too short (e.g. two months instead of three). It is worth noting that acute medication overuse (MO) was either 


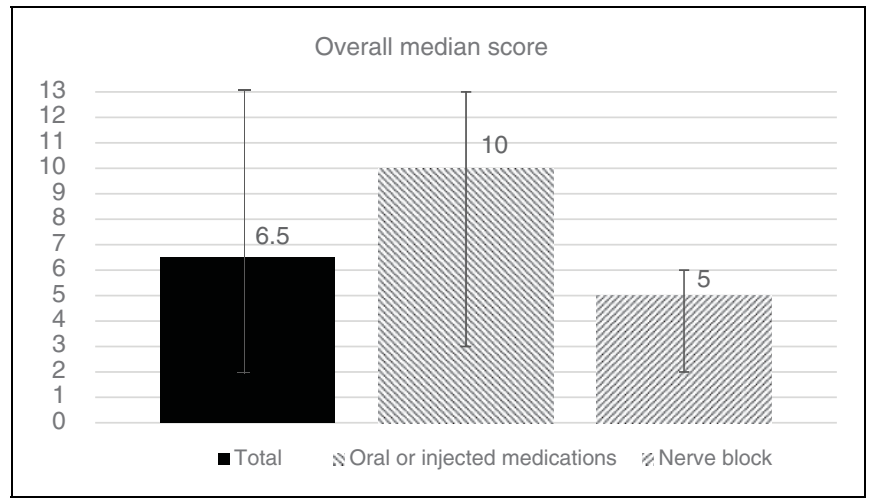

Figure 2. Median score (with range) of all papers evaluated (black bar), the score of papers dealing with oral or injected drugs and the score of drugs administered via nerve block.

Table 3. Adherence of the studies to the 2008 Guidelines.

\begin{tabular}{llll}
\hline & Oral or injected medications & Nerve blocks & Total \\
\hline General features & & $100 \%$ & $100 \%$ \\
Randomization at trial entry & $100 \%$ & $100 \%$ & $100 \%$ \\
Double-blind & $81.8 \%$ & $0 \%$ & $87.5 \%$ \\
Placebo-controlled & $72.7 \%$ & $0 \%$ & $31.25 \%$ \\
An $a$ priori defined outcome & $45.5 \%$ & $25 \%$ & $37.5 \%$ \\
NCT registration before recruitment & $54.5 \%$ & $20 \%$ & $30 \%$ \\
Migraine-specific features & & $60 \%$ & $37.5 \%$ \\
Chronic migraine according to ICHD-2 CM-R & $63.6 \%$ & $0 \%$ & $37.5 \%$ \\
Primary endpoint according to Guidelines & $45.5 \%$ & $63.6 \%$ & \\
Concomitant drug use & $63.6 \%$ & & \\
Treatment duration (3 months) & & & \\
\hline
\end{tabular}

not allowed or allowed and stratified for in only two thirds $(62.5 \%)$ of the trials.

As regards the success of blinding, it was impossible to correctly evaluate because most of the trials described insufficiently, or did not describe at all, the modality of assessment. The results are summarized in Table 3.

We attempted an evaluation of the effect size of the medications under investigation based on the level of adherence to the Guidelines, but this exercise proved uninformative due to the disparate adoption of primary endpoints in the various trials, the different classes of drugs tested, and the different routes/modalities of administration.

\section{Discussion}

Adherence to recommendations for the planning and conduct of clinical trials enhances the validity of the trials while the low adherence to migraine-specific criteria leads to heterogeneity across trials, which hampers a direct comparison. In this review of the literature, we found that most studies indeed followed the trial recommendations regarding the study design (randomization, double-blind, placebo-controlled), but this satisfying level of adherence was of course influenced by the search strategy that we adopted, which included the terms "randomized" and "controlled". When examining instead the specific recommendations for migraine trials or the recommendations regarding the end points, the adherence was much lower.

Only half of the studies used the recommended ICHD-2 CM-R criteria for the definition of CM. According to these, $\mathrm{CM}$ is defined as $\geq 15$ headache days per month of which $\geq 8$ fulfill criteria for migraine (25). This definition was an evolution of the original ICHD-2 definition of CM ( $\geq 15$ migraine days/month) 
(26), which was deemed insufficient to correctly identify $\mathrm{CM}$ within the larger group of chronic headaches. Consequently, the results of the trials using ICHD-2 cannot be fully generalized to the CM population.

The definition of primary endpoints is crucial for proper comparison of efficacy between migraine preventives. In our analysis, the primary endpoints varied greatly among studies and only one third followed the guideline recommendations. Furthermore, some trials failed to clearly specify primary versus secondary endpoints; the primary endpoint was often not clearly defined. This diverse selection and description of endpoints prevents the comparison of efficacy of different drugs across trials, which is exactly our experience in this analysis. The possibility of calculating the effect size of interventions is extremely important, since efficacy has been rated by migraine patients as the most important feature of a preventive drug (27). Single trials are essential for defining efficacy and tolerability of new therapeutic options. However, because of their nature, RCTs have intrinsically a low generalizability since they are conducted on a very selected population and according to strict rules (28). The comparison of multiple trials, conducted on diverse CM populations (e.g. patients refractory or naïve to treatments, with or without psychiatric comorbidity) provides a higher degree of generalizability, if the studies are comparable. Full adherence to the recommendations is likely the best way to ensure methodological consistency and therefore comparability.

When testing a new drug candidate, both randomization and blinding are essential components of the design of the clinical trial. Here, all trials were randomized and almost $90 \%$ were double-blinded. However, in most studies the randomization method was not described and, more importantly, it was not reported whether blinding was successful. Assessment of blinding success is mandatory in order to evaluate the true efficacy of the drug; for example, both the placebo effect and treatment effect may be more trustworthy if patients were sufficiently blinded. Since the placebo response is generally high in migraine patients (29), it is especially relevant to include placebo, and to preserve blinding in clinical trials in migraine. In this frame, it is surprising that some trials were not placebo controlled: three trials compared two active drugs used for preventive treatment in CM. This approach appears correct for the comparison of the effect size of drugs with proven efficacy but it may not be the best choice for evaluating the efficacy of a new drug candidate, especially in a field where prophylactic drugs with proven efficacy are limited, blinding can be unmasked by the side effects, and the drugs' effect size is limited.

Chronic migraine patients are often difficult to treat, and some may even have refractory headaches (30).
Thus, it may be necessary or appropriate to include patients who already take a preventive medication. The adherence to the recommendations on concomitant preventive drugs was quite high, though far from the ideal $100 \%$, as the majority of trials $(62.5 \%)$ either applied monotherapy or applied add-on therapy with a timing congruous with the Guideline recommendations. When evaluating efficacy of drugs and choosing the right treatment for the patients, it is important to distinguish between these two kinds of trials since addon therapy may provide a therapeutic gain for some patient groups but not for all.

Another important aspect to consider when dealing with $\mathrm{CM}$ patients is the presence of medication overuse (MO). MO plays an important role in the development of $\mathrm{CM}$ and removal of MO improves headache in a large proportion of patients (31-33). Stratification for $\mathrm{MO}$, or, at very least, a specified approach to MO during the trial, is very important since patients may become aware of the importance of reducing acute medications during the trial, which will ultimately prevent disentangling of the effect of the reduction in the intake of acute medications from the effect of the preventive drug.

It is not clear why certain trials followed some parts of the Guidelines but not others. This may depend on logistics, financing, or practical issues. For example, nerve block studies had a shorter duration of treatment (less than the recommended 3 months) compared to other studies. It may also depend on priority issues of the trial sponsor. Geography of regulatory requirement may be another source of variation (34).

The findings of this review have limitations. Firstly, we used a simplified version of the Guidelines to assess adherence. We based our scoring on 13 recommendations, which were considered to be of the highest importance for the evaluation of the quality of the trials. The hierarchy was discussed and agreed upon within the group of authors, building on a previous similar experience conducted on trials for episodic migraine (8). We acknowledge that other recommendations of the Guidelines could have been included as well. Secondly, we gave equal weight to each of the 13 items of the scoring system. We acknowledge that this can lead to two studies having similar scores but being of completely different quality depending on which criteria they followed. However, depending on the objective of the trial, which items should be considered more important may differ, and due to the large variation between studies we did not find it plausible to differentiate between the recommendations in our study. Therefore, we also chose not to determine a cut-off for an acceptable score. Thirdly, the trials were evaluated based on the description of the trial in 
the published paper, or in its supplementary materials, or on the relative page at clinicaltrials.gov. Some papers were unclear in the description of endpoints or period of observation, or other parts. Whenever a clear description was missing, we scored 0 for the corresponding item. This may have resulted in lower scores for some papers and directly impacted the validity and reliability of inferences drawn from the scores. In addition, if the findings of a single trial are split over different papers, separately reporting different outcomes, this will result in a lower score for one or more of the single papers. This underlines the importance of transparency and thorough descriptions when publishing papers on trials.

It is worth noting that, though imperfectly following the Guidelines, the RCTs conducted in the decade 2008-2017 have been very helpful and informative for elaborating the new set of Guidelines (7). These latter built on the 2008 edition to improve and expand some pivotal areas (Supplemental Table 1): This is the case for primary and secondary end-points, which are more clearly listed and defined, and the duration of disease, which has been extended to 12 months to minimize the occurrence of spontaneous reduction of attacks. In addition, the 2018 Guidelines provide precise indications for the management of critical issues, such as MO.

\section{Conclusion}

We assessed the adherence of CM clinical trials conducted since 2008 to the Guidelines for controlled trials of preventive treatment of chronic migraine in adults published in the same year and found that, while most studies followed the general recommendations for study design, the migraine-specific and endpoint-related recommendations were followed to a lesser extent. This diversity hampers comparison of results across trials for CM preventive treatments.

Guidelines on clinical trials are an extremely important tool to ensure not only the uniformity and quality of clinical trials, but also the subsequent steps: Availability on the market of effective drugs, scientific advances, optimized management, change in healthcare approaches, and so on. This is the reason why IHS undertook the effort to release in 2018 a new version of the Guidelines (7), improved and guided by the experience gathered in 10 years of RCTs. It is expected that investigators will carefully follow these new guidelines to the greatest possible extent.

Future studies on preventive treatments in $\mathrm{CM}$ should carefully follow the newly published updated migraine clinical trial guidelines. This will enhance the external validity and quality of the trials, as well as improve the evidence-based treatment for CM patients.

\section{Article highlights}

- Recently published clinical trials showed limited adherence to the 2008 Guidelines of the International Headache Society (IHS) for controlled trials on preventive treatment of chronic migraine in adults.

- Most studies followed the general IHS recommendations for study design, while the migraine-specific and end-point-related recommendations were followed to a lesser extent.

- This diversity hampers comparison of results across trials.

- Evidence and experience accumulated support the need for the publication of an updated edition of Guidelines and for a greater adherence to these.

\section{Declaration of conflicting interests}

The authors declared the following potential conflicts of interest with respect to the research, authorship, and/or publication of this article: Marie Deen, Daniele Martinelli and Judith Pijpers have no conflicts of interest.

HC Diener received honoraria for participation in clinical trials, contribution to advisory boards or oral presentations from: Addex Pharma, Alder, Allergan, Almirall, Amgen, Autonomic Technology, AstraZeneca, Bayer Vital, Berlin Chemie, Böhringer Ingelheim, Bristol-Myers Squibb, Chordate, Coherex, CoLucid, Electrocore, GlaxoSmithKline, Grünenthal, Ipsen, Janssen-Cilag, Labrys Biologicals, Lilly, La Roche, 3M Medica , Medtronic, Menerini, Minster, MSD, Neuroscore, Novartis, Johnson \& Johnson, Pierre Fabre, Pfizer, Schaper and Brümmer, Sanofi, St. Jude, Teva and Weber \& Weber. Financial support for research projects was provided by Allergan, Almirall, AstraZeneca, Bayer, Electrocore, GSK, Janssen-Cilag, MSD and Pfizer. Headache research at the Department of Neurology in Essen is supported by the German Research Council (DFG), the German Ministry of Education and Research (BMBF) and the European Union. H.C. Diener has no ownership interest and does not own stocks of any pharmaceutical company. HCD serves on the editorial boards of Cephalalgia and Lancet Neurology. HCD chairs the Clinical Guidelines Committee of the German Society of Neurology and is a member of the Clinical Trials Committee of the IHS.

Stephen Silberstein has received honoraria from Alder Biopharmaceuticals; Allergan, Inc.; Amgen; Avanir Pharmaceuticals, Inc.; Cefaly Technology (unrelated to this manuscript); Depomed; Dr. Reddy's Laboratories; eNeura 
Inc.; electroCore Medical, LLC; Ipsen Biopharmaceuticals; Medscape, LLC; Medtronic, Inc.; Mitsubishi Tanabe Pharma America, Inc.; NINDS; St. Jude Medical; Supernus Pharmaceuticals, Inc.; Teva Pharmaceuticals and Trigemina, Inc.

Michel D Ferrari reports grants and consultancy or industry support from Medtronic and independent support from the European Community, NWO, NIH and the Dutch Heart Foundation.

Messoud Ashina is a consultant, speaker or scientific advisor for Allergan, Amgen, Alder, ATI, Eli Lilly, Novartis, and Teva, primary investigator for Amgen 20120178 (Phase 2), 20120295 (Phase 2), 20130255 (OLE), 20120297 (Phase 3), Alder ALD403-CLIN-001 (Phase 3), Amgen PAC1 20150308 (Phase 2 a), and GM-11 gamma-Core-R trials.

Cristina Tassorelli has received consulting fees from Allergan, Eli-Lilly, ElectroCore, Novartis and Teva. She is PI in clinical trials sponsored by Allergan, Eli-Lilly, ElectroCore, Novartis and Teva.

Dr. Yuan received honoraria from Supernus Pharmaceuticals, Inc.

\section{Declaration of conflicting interests}

The authors declared no potential conflicts of interest with respect to the research, authorship, and/or publication of this article.

\section{Funding}

The authors received no financial support for the research, authorship, and/or publication of this article.

\section{ORCID iD}

Marie Deen (D) https://orcid.org/0000-0001-7818-9841

Daniele Martinelli (D https://orcid.org/0000-0001-6355-7947

Cristina Tassorelli (D https://orcid.org/0000-0003-1513-2113

\section{References}

1. Skripka-Serry J. The great neuro-pipeline 'brain drain' (and why Big Pharma hasn't given up on CNS disorders). Drug Discovery World 2013; 14: 9-16.

2. Olesen J, Bousser MG, Diener HC, et al. New appendix criteria open for a broader concept of chronic migraine. Cephalalgia 2006; 26: 742-746.

3. Silberstein S, Tfelt-Hansen P, Dodick DW, et al. Guidelines for controlled trials of prophylactic treatment of chronic migraine in adults. Cephalalgia 2008; 28: 484-495.

4. International Headache Society Committee on Clinical Trials in Migraine. Guidelines for Controlled Trials of Drugs in Migraine. First Edition. Cephalalgia 1991; 11: $1-12$.

5. International Headache Society Committee on Clinical Trials in Tension-type Headache. Guidelines for trials of drug treatments in tension-type headache. Cephalalgia 1995; 15: 165-179.

6. International Headache Society Committee on Clinical Trials in Cluster Headache. Guidelines for controlled trials of drugs in cluster headache. Cephalalgia 1995; 15: 452-462.

7. Tassorelli C, Diener HC, Dodick DW, et al. Guidelines of the International Headache Society for controlled trials of preventive treatment of chronic migraine in adults. Cephalalgia 2018; 38: 815-832.

8. Hougaard A and Tfelt-Hansen P. Are the current IHS guidelines for migraine drug trials being followed? J Headache Pain 2010; 11: 457-468.

9. Silberstein SD, Dodick DW, Lindblad AS, et al. Randomized, placebo-controlled trial of propranolol added to topiramate in chronic migraine. Neurology 2012; 78: 976-984.

10. Soares A, de A, Louçana PMC, Nasi EP, et al. A doubleblind, randomized, and placebo-controlled clinical trial with omega-3 polyunsaturated fatty acids (OPFA $\omega-3$ ) for the prevention of migraine in chronic migraine patients using amitriptyline. Nutr Neurosci 2018; 21: 219-223.

11. Kwok YH, Swift JE, Gazerani P, et al. A double-blind, randomized, placebo-controlled pilot trial to determine the efficacy and safety of ibudilast, a potential glial attenuator, in chronic migraine. J Pain Res 2016; 9: 899-907.

12. Ebrahimi-Monfared M, Sharafkhah M, Abdolrazaghnejad A, et al. Use of melatonin versus valproic acid in prophylaxis of migraine patients: A doubleblind randomized clinical trial. Restor Neurol Neurosci 2017; 35: 385-393.

13. Lai KL, Niddam DM, Fuh JL, et al. Flunarizine versus topiramate for chronic migraine prophylaxis: A randomized trial. Acta Neurol Scand 2017; 135: 476-483.

14. Kashipazha D, Ghadikolaei H and Siavashi M. Levetiracetam in compare to sodium valproate for prophylaxis in chronic migraine headache: A randomized double-blind clinical trial. Curr Clin Pharmacol 2017; 12: $55-59$.

15. Bigal ME, Dodick DW, Rapoport AM, et al. Safety, tolerability, and efficacy of TEV-48125 for preventive treatment of high-frequency episodic migraine: A multicentre, randomised, double-blind, placebo-controlled, phase $2 \mathrm{~b}$ study. Lancet Neurol 2015; 14: 1081-1090.

16. Bigal ME, Dodick DW, Krymchantowski AV, et al. TEV-48125 for the preventive treatment of chronic migraine. Neurology 2016; 87: 41-48.

17. Silberstein SD, Dodick DW, Bigal ME, et al. Fremanezumab for the preventive treatment of chronic migraine. N Engl J Med 2017; 377: 2113-2122.

18. Tepper S, Ashina M, Reuter U, et al. Safety and efficacy of erenumab for preventive treatment of chronic migraine: A randomised, double-blind, placebo-controlled phase 2 trial. Lancet Neurol 2017; 16: 425-434.

19. Song JH, Zhang GB, Ding XD, et al. Efficacy of type a botulinum toxin injections and infrared polarized light on treating chronic migraine. Eur Rev Med Pharmacol Sci 2015; 19: 1976-1982.

20. Gul HL, Ozon AO, Karadas O, et al. The efficacy of greater occipital nerve blockade in chronic migraine: A placebo-controlled study. Acta Neurol Scand 2017; 136: $138-144$. 
21. Cuadrado ML, Aledo-SerranoÁ, Navarro P, et al. Shortterm effects of greater occipital nerve blocks in chronic migraine: A double-blind, randomised, placebo-controlled clinical trial. Cephalalgia 2017; 37: 864-872.

22. Dilli E, Halker R, Vargas B, et al. Occipital nerve block for the short-term preventive treatment of migraine: A randomized, double-blinded, placebo-controlled study. Cephalalgia 2015; 35: 959-968.

23. Palamar D, Uluduz D, Saip S, et al. Ultrasound-guided greater occipital nerve block: An efficient technique in chronic refractory migraine without aura? Pain Physician 2015; 18: 153-162.

24. Inan LE, Inan N, Karadaş Ö, et al. Greater occipital nerve blockade for the treatment of chronic migraine: A randomized, multicenter, double-blind, and placebo-controlled study. Acta Neurol Scand 2015; 132: 270-277.

25. Silberstein SD, Lipton RB and Sliwinski M. Classification of daily and near-daily headaches: Field trial of revised IHS criteria. Neurology 1996; 47: 871-875.

26. Headache Classification Subcommittee of the International Headache Society. The International Classification of Headache Disorders, 2nd edition. Cephalalgia 2004; 24: 9-160.

27. Peres MFP, Silberstein S, Moreira F, et al. Patients' preference for migraine preventive therapy. Headache 2007; 47: 540-545.
28. Rothwell PM. External validity of randomised controlled trials: "To whom do the results of this trial apply?" Lancet 2005; 365: 82-93.

29. Macedo A, Baños JE, Farré M, et al. Placebo response in the prophylaxis of migraine: A meta-analysis. Eur J Pain 2008; 12: 68-75.

30. Goadsby PJ, Schoenen J, Ferrari MD, et al. Towards a definition of intractable headache for use in clinical practice and trials. Cephalalgia 2006; 26: 1168-1170.

31. Munksgaard SB and Jensen RH. Medication overuse headache. Headache 2014; 54: 1251-1257.

32. Tassorelli C, Jensen R, Allena M, et al., on behalf of the COMOESTAS Consortium. The added value of an electronic monitoring and alerting system in the management of medication-overuse headache: A controlled multicentre study. Cephalalgia 2017; 37: 1115-1125.

33. Paemeleire K, Louis P, Magis D, et al. Diagnosis, pathophysiology and management of chronic migraine: A proposal of the Belgian Headache Society. Acta Neurol Belg 2015; 115: 1-17.

34. Alqahtani S, Seoane-Vazquez E, Rodriguez-Monguio R, et al. Priority review drugs approved by the FDA and the EMA: Time for international regulatory harmonization of pharmaceuticals? Pharmacoepidemiol Drug Saf 2015; 24: 709-715. 


\section{DuEPublico}

Duisburg-Essen Publications online
DE UISEBN R G

offen im Denken

Ub | $\begin{aligned} & \text { universitäts } \\ & \text { bibliothek }\end{aligned}$

This text is made available via DuEPublico, the institutional repository of the University of Duisburg-Essen. This version may eventually differ from another version distributed by a commercial publisher.

DOI: $\quad 10.1177 / 0333102419847751$

URN: urn:nbn:de:hbz:464-20210510-120704-5

This publication is with permission of the rights owner freely accessible due to an Alliance licence and a national licence (funded by the DFG, German Research Foundation) respectively.

(C) International Headache Society 2019. All rights reserved. 